
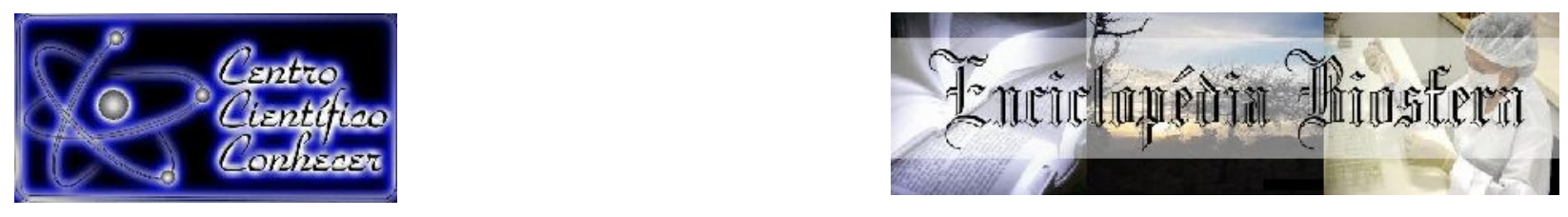

\title{
IMPLANTAÇÃO DE SISTEMA DE GERÊNCIA DE PAVIMENTOS: TRECHO DA BR-135
}

\author{
Jorcelan Pereira da Rocha ${ }^{1}$, Larysse Lohana Leal Nunes ${ }^{2}$
}

1 Engenheiro civil. Pós-graduando em Infraestrutura de Transportes e Rodovias pelo Instituto de graduação e pós-graduação - IPOG (jorcelan20@gmail.com) - São Luís, Maranhão, Brasil.

2 Engenheira civil. Pós-graduanda em Infraestrutura de Transportes e Rodovias pelo Instituto de graduação e pós-graduação - IPOG- São Luís, Maranhão, Brasil.

\section{Recebido em: 06/04/2019 - Aprovado em: 10/06/2019 - Publicado em: 30/06/2019 DOI: 10.18677/EnciBio_2019A180}

Este trabalho foi executado com a finalidade de mostrar que o Sistema de Gerência de Pavimentos (SGP) é essencial para uma melhor administração rodoviária, pois permite a previsão de gastos para cada solução a ser adotada e, consequentemente, menores custos aos cofres públicos. Para isso se realizou um estudo através da implantação de SGP para investigação e tomada de decisão em um trecho da BR-135 no estado do Maranhão. Nesta pesquisa se buscou definir quais os benefícios gerados por uma análise em nível de projeto para um segmento de 25,6 km através da comparação de diversas alternativas de intervenções. A coleta de dados para o estudo ocorreu por meio da cooperação da SRMA/DNIT. Esses dados, posteriormente, foram aplicados no programa HDM-4, gerando assim, resultados que consideraram 0 desempenho do pavimento em relação às estratégias de Manutenção e Restauração (M\&R) para um período de 20 anos. Entre as soluções propostas, a análise indicou que a melhor alternativa de intervenção é o CREMA 1. Além disso, possibilitou a verificação da diferença entre os valores de cada estratégia, permitindo fixar a solução ideal que esteja dentro da capacidade financeira da agência, não ocasionando resultados que posteriormente poderão ficar sem aplicabilidade. Por fim, se concluiu a eficiência do SGP, porque se percebe que foi um passo imenso no que diz respeito não só a obtenção de resultados, mas também na formação de um banco de dados que pode ser facilmente acessível e atualizado, admitindo novas de decisões no decorrer do tempo.

PALAVRAS-CHAVE: Estratégia.HDM-4. Sistema de gerência.

\section{IMPLEMENTATION OF PAVEMENT MANAGEMENT SYSTEM: SECTION OF BR-135}

\begin{abstract}
This work was carried out with the purpose of showing that the Pavement Management System (PMS) is essential for a better road administration, since it allows the forecasting of expenses for each solution to be adopted and, consequentily, lower costs to the public coffers. For that, a study was carried out
\end{abstract}


through the implementation of PMS for investigation and decision making in a section of BR-135 in the state of Maranhão. The purpose of this research was to define the benefits generated by a project-level analysis for a $25.6 \mathrm{~km}$ segment, by comparing several alternative interventions. Data collection for the study took place through the cooperation of SRMA/DNIT. These data were later applied to the HDM-4 program, generating results that considered the pavement performance in relation to Maintenance and Restoration (M\&R) strategies for a period of 20 years. Among the proposed solutions, analysis indicated that the best alternative of intervention is the CREMA 1. In addition, it made possible the verification of the difference between the values of each strategy, allowing to fix the ideal solution that is within the financial capacity of the agency, not causing in results that may later become inapplicable. Finally, the efficiency of the PMS was concluded, because it is perceived that it was an immense step not only in obtaining results, but also in the formation of a database that can be easily accessible and update, admitting new decisions over time.

KEYWORDS: Management System. HDM-4. Strategy.

\section{INTRODUÇÃO}

O transporte rodoviário é o principal modal de transporte no território brasileiro, sendo o responsável pela grande maioria das movimentações, sejam de carga $(61,1 \%)$ ou passageiros $(95 \%)$, tornando-se de fundamental importância em aspectos socioeconômicos (CNT, 2016). Apesar de sua grande relevância, não existe o devido cuidado no que diz respeito à aplicação de recursos em atividades relacionadas com esse modal, porquanto são poucos os estudos que enfatizam uma melhor administração rodoviária no território brasileiro (DNIT, 2011).

Dessa forma, muito se tem discutido, recentemente, acerca de uma boa gerência dos recursos públicos para o desenvolvimento do país, contudo, a degradação de rodovias contribui para que os recursos sejam desperdiçados em intervenções que não respeitam a relação de custo-benefício, pois, na maioria das vezes, as atividades de Manutenção e Restauração (M\&R) só acontecem quando as rodovias estão próximas de atingir seu limite de trafegabilidade. Além do desperdício financeiro gerado pela falta de planejamento na aplicabilidade dos recursos, rodovias com problemas funcionais, estruturais e operacionais podem ocasionar desconforto e insegurança ao usuário da via e, ainda, um impacto ambiental mais acentuado (CNT, 2018).

Conforme DNIT (2011) ao considerar tal imprudência cometida por agências rodoviárias em todo Brasil, destaca-se que decisões que analisam as devidas e prioritárias intervenções através de um banco de dados bem estruturado são possíveis por meio da implantação do Sistema de Gerência de Pavimentos (SGP), tendo por principal objetivo garantir um diagnóstico adequado que possa levar a intervenções predeterminadas, em conformidade com estudos prévios. Assim, o SGP reúne informações com objetivo desenvolver um processo contínuo e integrado de ações que obtenham o melhor resultado possível referente à tomada de decisão na administração de recursos que serão aplicados no setor rodoviário. Tais informações devem ser fisicamente localizadas em relação à rede rodoviária, formando um eficiente sistema de referência para a análise dos dados coletados. De forma resumida, o SGP é uma estrutura que está relacionada diretamente com fatores internos e externos que possibilitam a formação de um amplo banco de dados. Para isso, segundo ODA (2016), na coleta de informações em campo devem ser utilizados equipamentos que possibilitam a análise de grandes redes viárias em 
um curto espaço de tempo, como por exemplo: veículos que fotografam ou filmam os pavimentos.

Paralelamente, Hudson et al. (1979) destacam que o uso do Sistema de Gerência de Pavimentos pode ser de forma ampla ou subdividido em vários níveis de gestão, com isso, a preparação da tomada de decisão pode ocorrer para projetos individuais ou para uma rede rodoviária inteira. Devendo considerar todos os tipos decisões, inclusive aquelas que expõem a carência de informações, deficiências projetadas ou necessidades de melhoria para a rede como um todo, orçamentação, programação, pesquisa, projeto de projeto, construção e manutenção, requisitos de recursos, monitoramento, entre outras.

$\mathrm{Na}$ tomada de decisões relacionadas atividades de Manutenção e Restauração (M\&R) têm uma grande quantidade de métodos avaliativos, como: Método da Matriz, Árvore de Decisão, Método do Custo do Ciclo de Vida e Método de Otimização (ODA, 2016). Em virtude dessas características, DNIT (2011) define o SGP como um artifício que possibilita, no decorrer do tempo, a averiguação contínua da estrutura do pavimento (revestimento, base, sub-base e subleito) que fica submetida a fatores externos, como o tráfego, as operações de manutenção e as condições ambientais.

O Consórcio Dynatest Engemap (2015) adverte que o SGP, tecnicamente, é fundamentado em três diferentes modelos: Matrizes de soluções: encarregadas pela designação das intervenções que poderão ser empregadas com base no estado presente do pavimento; Estimativa de custos: com base nas intervenções designadas, necessita ser feito uma estimativa de investimento com a finalidade de aplicação no sistema de gerência; Previsão de desempenho: possibilita prever, com HDM-4, o desenvolvimento das condições funcionais, estruturais e a necessidade dos múltiplos trechos rodoviários, no qual, são considerados os indicadores atuais, tornando-se substancial para o planejamento.

A Figura 1 demonstra que os Sistemas de Gerência de Pavimentos, em regra, podem ser classificados em dois níveis generalizados: (1) o nível de rede, onde são tomadas as decisões administrativas que atingem os programas para redes rodoviárias em geral, e (2) o nível de projeto, onde são feitas decisões técnicas para projetos específicos. No entanto, pode-se encontrar uma série de atividades técnicas no primeiro nível e decisões administrativas no segundo nível. Isto é, o SGP é composto por uma base de dados e as áreas de atividades são identificadas nos níveis de projeto e rede (HUDSON et al., 1979).

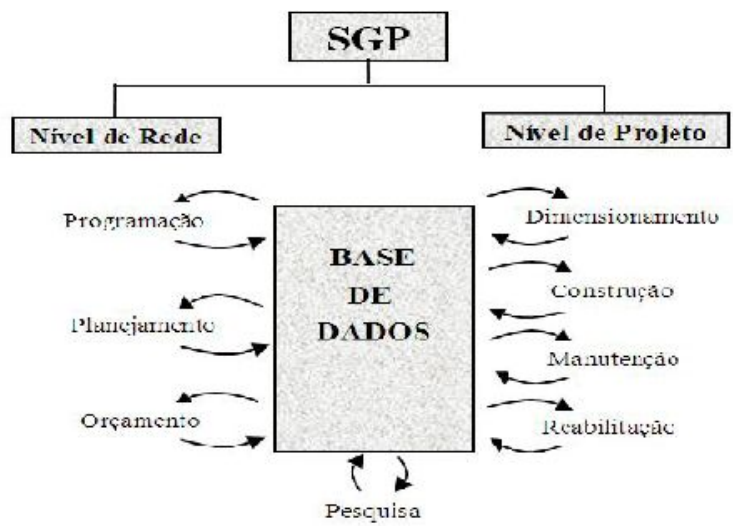

FIGURA 1 - Interação entre os níveis de decisão no SGP Fonte: Adaptada de HUDSON et al. (1979) 
Para ODA (2014) existem diversas atividades quem podem se encaixar dentro do nível de projeto, como por exemplo: Escolha dos serviços de manutenção, reabilitação e reconstrução, com base em critérios determinados no nível de rede; Fornecimentos de informação que atualizem a base de dados sobre projeto, construção e manutenção; Determinação dos parâmetros de projeto, como: resistência do subleito, número do eixo padrão e especificações para os materiais.

Deve-se destacar também, que as questões climáticas interferem diretamente nas condições dos pavimentos de asfalto. Assim, as diversas condições presentes na estrutura do pavimento são afetadas de diferentes formas e estão relacionadas diretamente com o desempenho do pavimento. Por conseguinte, é necessário utilizar modelos que capturam ao máximo o real comportamento material em termos de fatores climáticos, como temperatura e precipitação (ZOFKA, 2018).

Por fim, no estado maranhense, no que refere à busca por maior eficiência no controle de informações sobre as rodovias, verifica-se que ainda há muito que se desenvolver, porém, este trabalho representa um passo positivo quanto ao desenvolvimento do SGP neste Estado, pois tal estudo possibilita a obtenção de informações para um banco de dados que, posteriormente, podem ser aplicadas no software HDM-4 para obtenção de resultados que priorizam intervenções conforme as limitações do administrador rodoviário.

\section{MATERIAL E MÉTODOS \\ Especificações e localização geográfica do trecho em estudo}

Esta pesquisa foi efetivada através da SRMA/DNIT que possibilitou a transformação de informações em dados com o objetivo de mostrar a viabilidade e utilidade de decisões usando o Sistema de Gerência de Pavimentos em um segmento da BR-135/MA, sendo de fundamental importância para o território maranhense, pois é a única rodovia que liga a capital do Maranhão (São Luís) com o restante do país, especificadamente, vai de São Luís-MA até Belo Horizonte MG.

Detalhadamente, o trecho em estudo se caracteriza por:

a) Rodovia: BR-135/MA

b) Trecho: Santa Rita - Entrada da BR-222(A) (Pov: Outeiro)

c) Segmento: $\mathrm{km} \mathrm{70,0}$ ao $\mathrm{km} \mathrm{95,6}$

d) Extensão: 25,6 km

e) Código PNV: 135BMA0080

Na Figura 2, é possível observar detalhadamente a localização do segmento estudado.

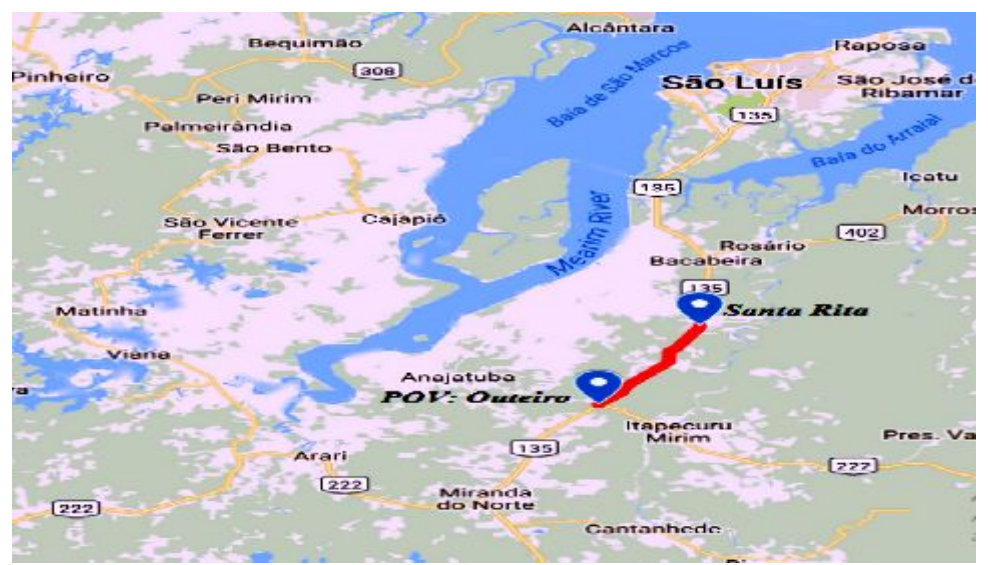

FIGURA 2 - Trecho da BR-135 em estudo

Fonte: Adaptada de Google Maps 
A Figura 3 demonstra o pavimento asfáltico analisado.

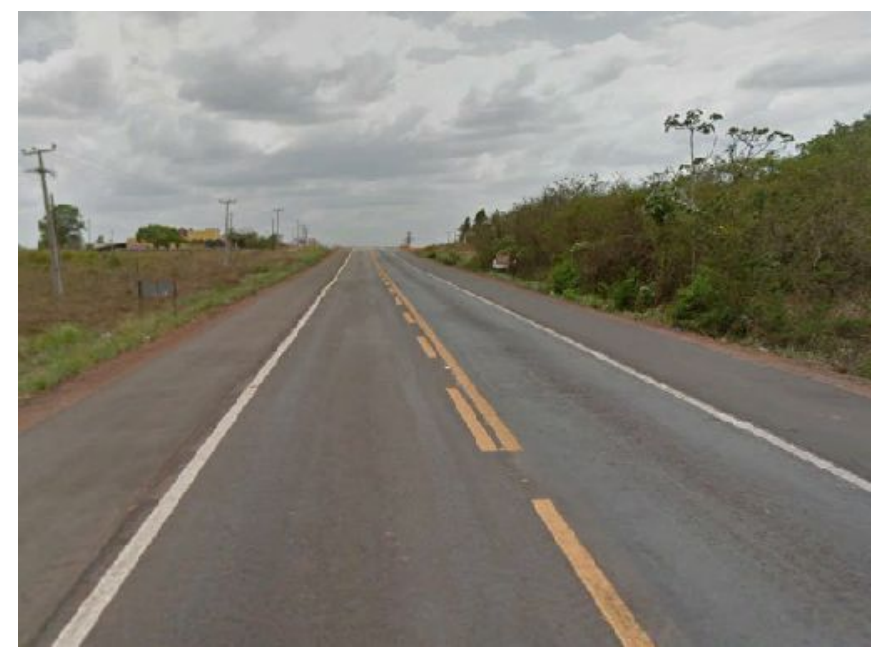

FIGURA 3 - Pavimento asfáltico do trecho em estudo Fonte: Elaborada pelos autores (2018)

\section{Programa utilizado na pesquisa}

Para processamento das informações foi utilizado o programa HDM-4, que segundo Kerali (2000) é a ferramenta mais desenvolvida mundialmente para auxiliar engenheiros na formulação de um Sistema de Gerência de Rodovias, especialmente no que se refere a verificações econômicas de alternativas de investimento. Essa verificação só é possível, pois o software é capaz de reunir uma grande quantidade de informações sobre o modo de transporte (condições da rodovia, custos de manutenção, custo de operação, etc). Para obtenção de resultados precisos, o programa leva em consideração o carregamento gerado pelo tráfego, condições climáticas e efeitos causados por um sistema de drenagem debilitado.

Consócio Dynatest Engemap (2015) alega que através do programa é possível à análise dos dados via intranet, acatando as regras de segurança da informação através de níveis de acesso do usuário cadastrado, centralizando o banco de dados, mas universalizando a informação, permitindo que cada especialista desenvolva consultas e exames referentes a um trecho ou à malha.

Em vista disso, com o HDM-4 é possível também realizar a priorização de investimentos (modelagem econômica), recomendando ao gestor os trechos que devem ser priorizados, levando em consideração a melhor forma de retorno econômico, associado às estimativas de investimento das intervenções previstas. Sendo recomendada para remodelagem do SGP uma matriz de planejamento gerencial, devidamente compatibilizada com as diretrizes de projeto do órgão, que permite definir o tipo e custo dos serviços e obras de manutenção nos segmentos viários da malha federal. Com o manuseio das informações no software HDM-4, um relatório é gerado para que a melhor opção de intervenção seja tomada pelo órgão.

Um dos principais resultados obtidos no programa é o Índice de irregularidade internacional (IRI), pois de acordo com o DNIT (2011), o IRI é um índice estático, expresso em $\mathrm{m} / \mathrm{km}$, utilizado para medir a irregularidade longitudinal de um pavimento por meio do somatório dos desvios da superfície em relação a um plano de referência ideal de projeto geométrico, relacionado diretamente com a dinâmica dos veículos, o efeito dinâmico das cargas, a qualidade ao rolamento e a drenagem superficial da via, portanto, através do IRI é possível quantificar os desvios da superfície do pavimento existente. 
Em conformidade com Kerali (2000), a estrutura analítica do software HDM-4 é constituída por três grandes blocos:

a) Gerenciador de dados: possibilita ao gerenciador determinar as características das condições a serem modeladas;

b) Ferramentas de análise: é composta por três formas de aplicação do programa, que são as análises por projetos, programas e estratégia;

c) Modelos: são a base matemática que embasam os cálculos e as análises concretizadas para aquisição dos efeitos sobre a qualidade da rodovia, custos dos usuários e impactos ambientais.

Portanto, essa estrutura analítica poder ser observada na Figura 4.

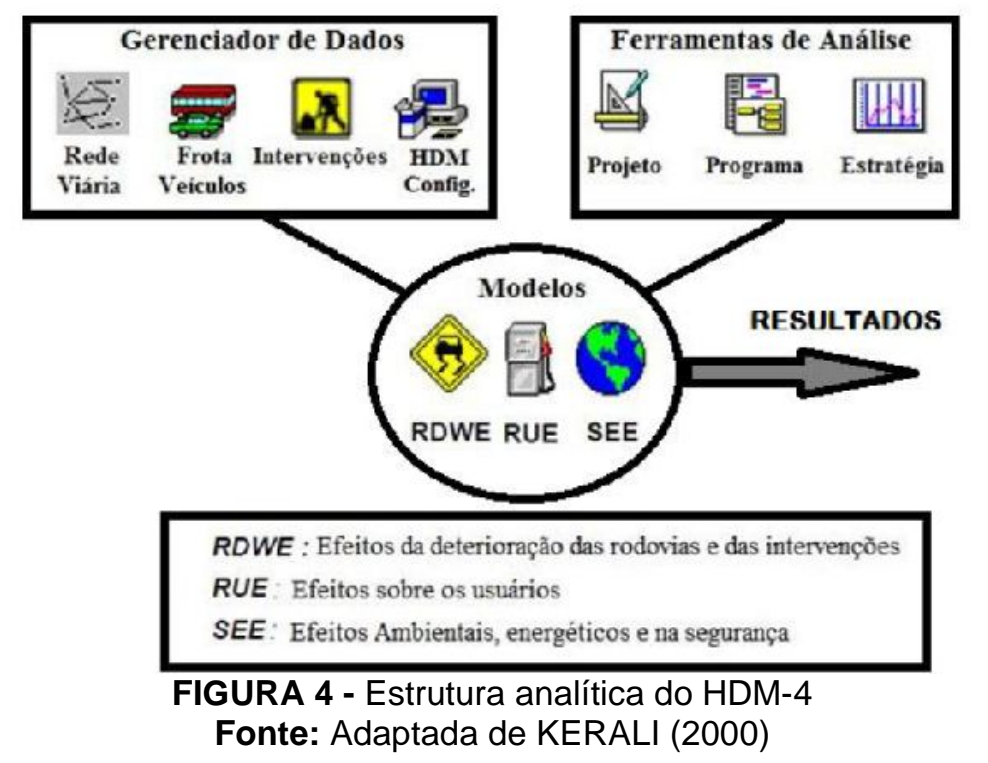

A calibração do HDM-4 depende muito do órgão e da finalidade desejada para aplicação do software no setor rodoviário. Assim, conforme Bennett e Paterson (2000), o programa é capacitado para lidar com diferentes níveis de tecnologia, clima e ambiente econômico, respeitando as características locais. Ou melhor, para execução de modelos no programa é necessário atenção nos seguintes passos: Aquisição dos dados de entrada: analisar perfeitamente os dados de entrada que são requisitados, interpretando-os de forma minuciosa para obtenção de informações em conformidade com o aproveitamento desejado; Calibração dos coeficientes: regular os coeficientes dos modelos com o objetivo de que as previsões e resultados sejam alcançados de acordo com a realidade vivenciada no local.

Os dados de entrada responsáveis pela obtenção de bons resultados no HDM-4 representam as características físicas do pavimento e da rede por completo, como exemplos têm os dados sobre os usuários, sobre o tráfego, sobre os custos unitários, sobre a economia. Acompanhado com o HDM-4, o Excel é utilizado como uma ferramenta para armazenamento e organização de informações, tornando-se como uma base para bancos de dados, sendo um programa de simplificada compreensão e utilização e, ainda, de fácil acesso a empresas e órgãos públicos (FERNANDES, 2017). 
RESULTADOS

O primeiro passo do estudo foi à coleta de informações durante alguns anos, gerando-se um banco de dados correspondentes à realidade do segmento. No decorrer do armazenamento foram feitas simulações, o que ocasionou diversos resultados descartados, pois se tornavam defasados com os novos elementos obtidos em campo. Com isso, verificou-se que quanto maior a número de dados inseridos no programa, melhor a precisão da análise.

Destaca-se também, que o estudo tem o inicio do período de análise em 2017, entretanto, o armazenamento considera informações desde 2010, como comprova os itens apresentados a seguir.

\section{Dados de entrada usados para análise no HDM-4}

a) Informações de ordem geral;

- Moeda (Currency): Real (Milhões);

- Ano de início do período de análise: 2017;

- Período de análise: 20 anos;

- Taxa de desconto (Discount rate): 12,00\%;

- Tipo de análise: Análise por projeto.

b) Janela definição (Definition);

- Nome da seção (Section Name): FIM PISTA DUPLA (SANTA RITA) ENTR BR-222(A) (OUTEIRO);

- Número de identificação da seção (Section ID): 135BMA0080;

- Tipo de fluxo (Speed flow type): Rodovia padrão de duas faixas (Two

Lane Standard);

- Tipo de fluxo de tráfego (Traf Flow Pattern): Fluxo Livre (Free Flow);

- Tipo de clima (Climate zone): Tropical Úmido (Tropical Humid);

- Classe da rodovia (Road Class): Primária ou Tronco (Primary or Trunk);

- Tipo de superfície (Surface class): Pavimento betuminoso (Bituminous);

- Tipo de pavimento (pavement type): Concreto asfáltico sobre base

granular (Asphalt mix on granular base);

- Extensão (Length): 25,6 km;

- Largura da pista (Carriageway width): 7,2 m;

- Largura do acostamento (Shoulder width): 2,0 m;

- Número de faixas (Number of Lanes): 2;

- VMD (Motorised AADT): 17683;

- Ano da última selagem, recapeamento ou restauração (Last surfacing):

2016;

- Sentido do tráfego (Traffic flow direction): Dois sentidos (two-way).

$\mathrm{Na}$ Figura 5, verifica-se a aplicação dos dados na janela definição. 


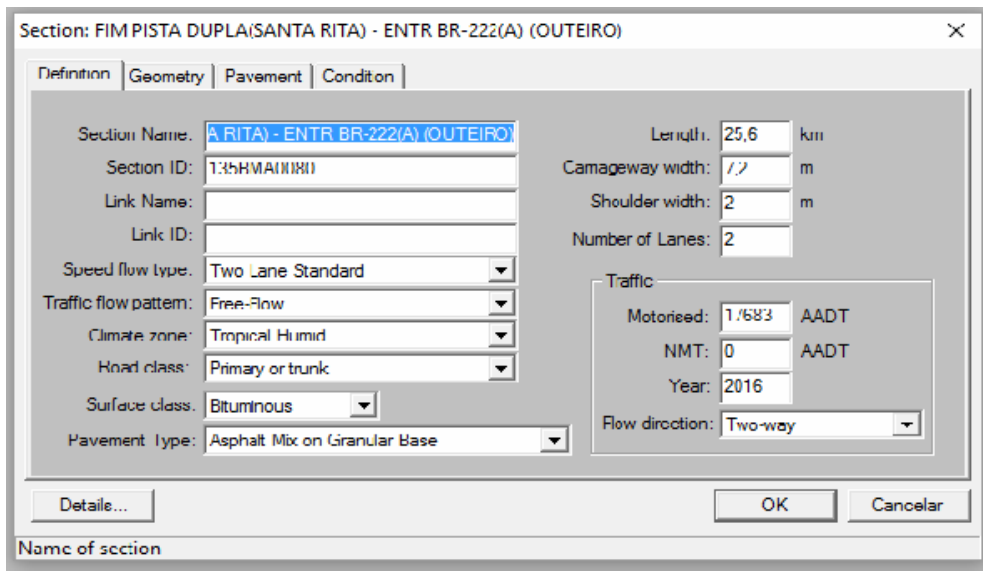

FIGURA 5 - Janela definição do programa HDM-4

Fonte: Captura de tela do software HDM-4 (2018)

c) Janela geometria (Geometry);

- Subidas e descidas (Rise+Fall): 1,00 m/km;

- Grau de curvatura (Avg horiz curvature): 3,00 deg/km;

- Velocidade limite (Speed limit): $110 \mathrm{~km} / \mathrm{h}$;

- Altitude: 43 m.

d) Janela pavimento (Pavement);

- CBR médio do subleito do segmento (Subgrade CBR): 4\%;

- Espessura de superfície mais recente (Most recent surfacing thickness):

$25 \mathrm{~mm}$;

- Espessura de superfície anterior (Previous/old surfacing thickness): 100 $\mathrm{mm}$;

- Ano da última construção/reconstrução (Last reconstruction or new construction): 2010;

- Ano da última reabilitação (Last rehabilitation): 2015;

- Ano da última resselagem (last resurfacing): 2015;

- Ano do último tratamento preventivo (last preventative treatment): 2015.

Na Figura 6, é possível observar de forma detalhada a aplicação dos dados na janela pavimento.

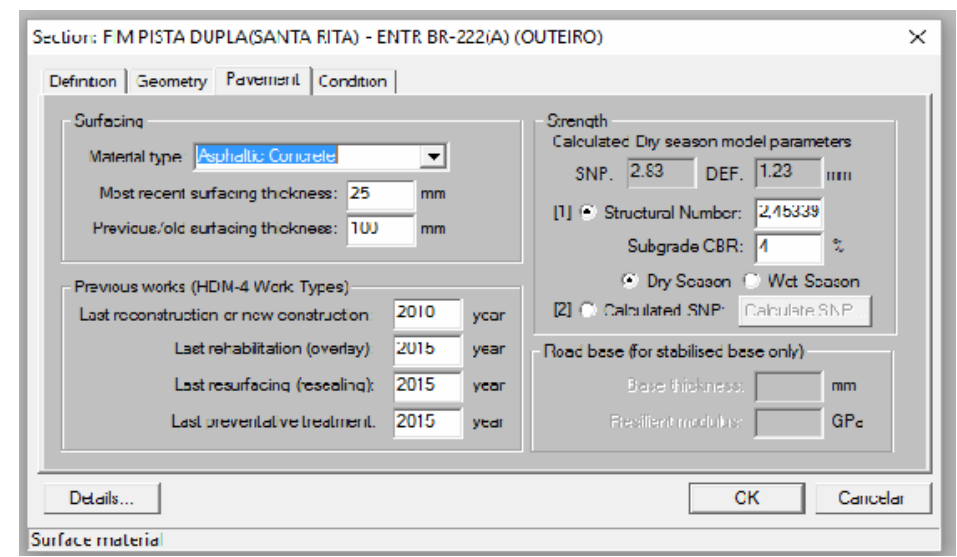

FIGURA 6 - Janela pavimento do programa HDM-4

Fonte: Captura de tela do software HDM-4 (2018)

e) Janela condição (Condition);

- Condições no final do ano (Condition at end of year): 2015;

- Irregularidade (Roughness - IRI): 6,0 m/km; 
- Área de trincamento (Total área of cracking): $15 \%$;

- Área de desgaste (Revelled área): $20 \%$;

- Número de buracos (Number of potholes): 5/km;

- Área de bordo quebrada (Edge break área): $100 \mathrm{~m}^{2} / \mathrm{km}$;

- Trilha de roda média (Mean rut depth): $15 \mathrm{~mm}$;

- Profundidade da textura (Texture depth): 0,30 mm;

- Resistência à derrapagem (Skid resistance - SCRIM $50 \mathrm{~km} / \mathrm{h}$ ): 0,35;

- Drenagem (Drainage): Ruim (Poor).

A Figura 7 destaca a aplicação dos dados na janela condição.

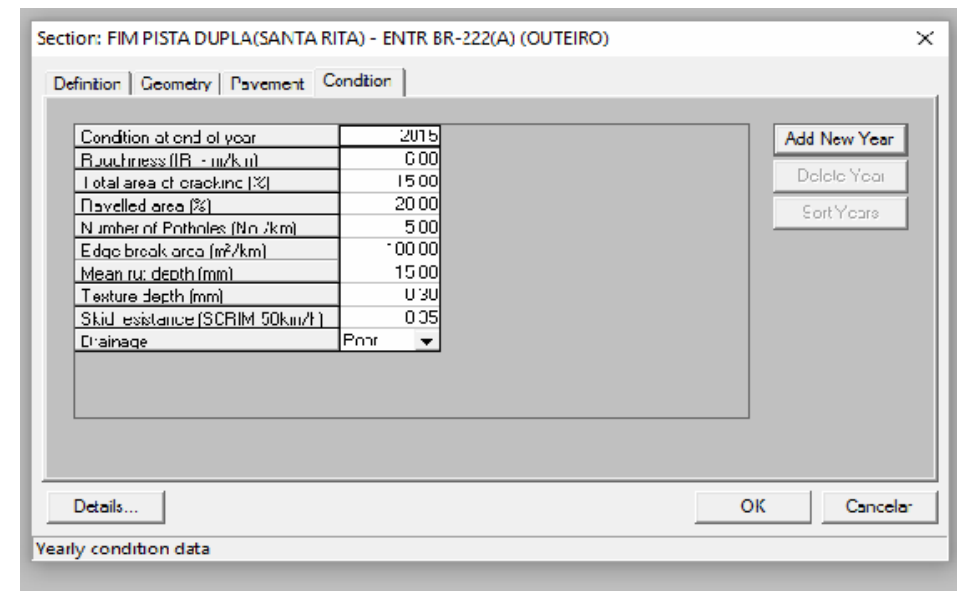

FIGURA 7 - Janela condição do programa HDM-4

Fonte: Captura de tela do software HDM-4 (2018)

f) Frota de veículos;

A Figura 8 mostra a frota de veículos que utilizam o trecho analisado.

HDM-4 - [Project: Análise Seção Santa Rita_Outeiro_BR 135]

Workspace View Report/Chart Window Help

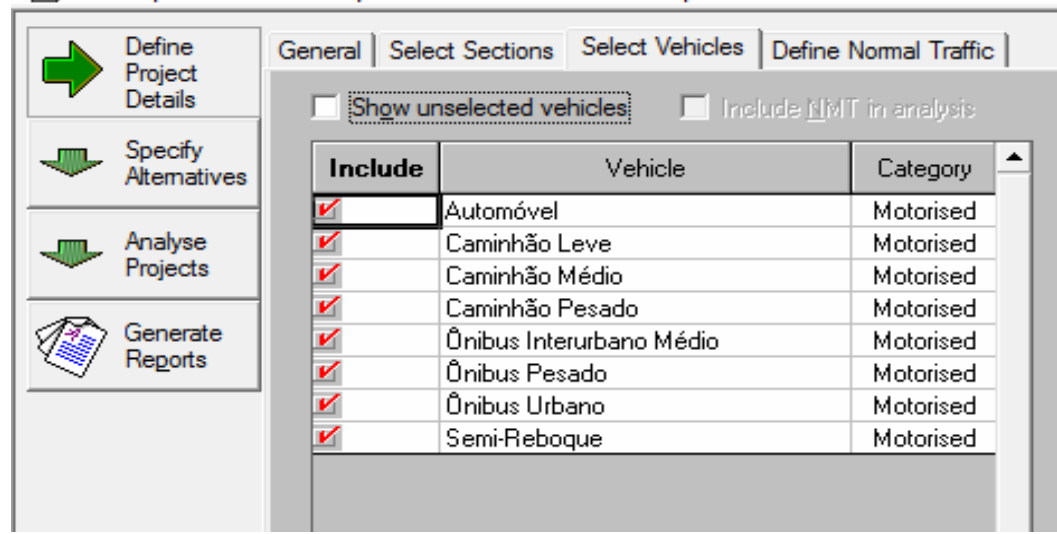

FIGURA 8 - Frota de veículos que utilizam a rede

Fonte: Captura de tela do software HDM-4 (2018)

Na Figura 9, estão exibidas em termos percentuais, a composição inicial dos veículos e suas taxas de crescimento anual a partir de 2017. 


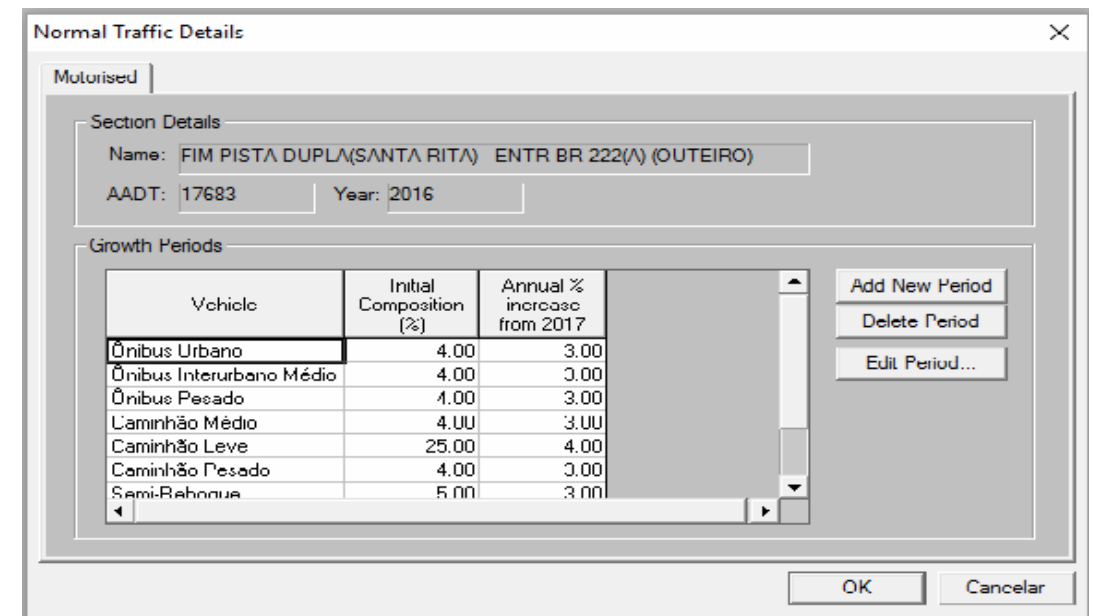

FIGURA 9 - Detalhes sobre a frota de veículos que utilizam a rede Fonte: Captura de tela do software HDM-4 (2018)

g) Especificações das alternativas adotadas;

- Alternativa base (serviços de conservação rotineira de pista simples):

deve ocorrer periodicamente; e seu custo médio é de 51.100,00 R\$/km;

Por se tratar de conservação periódica, essa alternativa ocorre anualmente, independente da realização de qualquer outra intervenção.

- CREMA 10 ETAPA: deve ocorrer quando o valor do trincamento estiver $\geq 1,0 \%$ e $\leq 50,0 \%$; e seu custo médio é $308.000,00 \mathrm{R} \$ / \mathrm{km}$;

- CREMA 20 ETAPA: deve ocorrer quando o afundamento de trilha de roda estiver $\geq 5,0 \mathrm{~mm}$ e $\leq 20,0 \mathrm{~mm}$; e seu custo médio é $625.000,00 \mathrm{R} \$ / \mathrm{km}$;

- Restauração: deve ocorrer quando o valor do IRI estiver $>3,0 \mathrm{~m} / \mathrm{km}$; e seu custo médio é 1.174.000,00 R $\$ / \mathrm{km}$ dividido em duas etapas (60\% e $40 \%$ );

- Reconstrução: deve ocorrer quando o número de panelas estiver $>3,0$ $/ \mathrm{km}$; e seu custo médio é $2.357 .000,00 \mathrm{R} \$ / \mathrm{km}$ dividido em duas etapas $(60 \%$ e $40 \%)$.

OBS: As informações (custos médios/serviços) supracitadas foram obtidas com o uso da tabela de custos médios gerenciais do DNIT.

Após a aplicação dos dados/critérios no programa HDM-4, foi realizada a simulação para classificar as alternativas de acordo com a melhor relação de custobenefício, tais resultados foram gerados por meio de gráficos e planilhas. Apesar de o estudo ser uma aplicação básica, a análise dos resultados mostrou que existe uma grande diferença de valores no que diz respeito às intervenções (alternativas de M\&R) para um período de 20 anos.

Sendo assim, na Figura 10 é possível observar de forma detalhada o resultado dos valores demonstrados como custos totais de transporte (sem taxa de desconto) e comprovar que as alternativas que geram menores custos podem ser classificadas na ordem decrescente da seguinte maneira: (1ํ) CREMA 1, (2º)

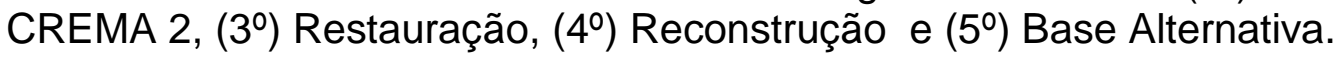




\section{Summary of Total Undiscounted Economic Costs by Alternative and Section:}

\begin{tabular}{|c|c|c|c|c|c|}
\cline { 2 - 6 } \multicolumn{1}{c|}{} & $\begin{array}{c}\text { Base } \\
\text { Alternative }\end{array}$ & CREMA 1 & CREMA 2 & Reconstru & RESTAURA \\
\hline $\begin{array}{c}\text { FIM PISTA DUPLA } \\
\text { (SANTA RITA) - ENTR } \\
\text { BR-222(A) }\end{array}$ & $17.640,02$ & $10.433,72$ & $10.457,27$ & $10.499,38$ & $10.451,37$ \\
\hline
\end{tabular}

FIGURA 10 - Resumo dos custos totais de transporte (sem taxa de desconto) obtido no HDM-4 Fonte: Resultado da pesquisa (2018)

$\mathrm{Na}$ Figura 11 foram obtidos detalhadamente todos os custos da agência (DNIT) no decorrer dos 20 anos, assim, é possível perceber os investimentos necessários para cada ano do período analisado. Demonstrando que a economia inicial feita na solução base alternativa resultará na perda da eficiência estrutural e funcional do pavimento, e maiores custos de transporte durante os 20 anos de análise.

Summary of Total Annual Economic Costs

\begin{tabular}{|c|c|c|c|c|c|}
\hline & Base Alternative & CREMA 1 & CREMA 2 & ReconstrupÒo & RESTAURAA $+O$ \\
\hline 2017 & $1,308,160.00$ & $9,192,960.00$ & $17,308,160.00$ & $37,511,680.00$ & $19,340,800.00$ \\
\hline 2018 & 1,308,160.00 & $1,308,160.00$ & $1,308,160.00$ & $25,443,840.00$ & $13,329,920.00$ \\
\hline 2019 & 1,308,160.00 & $1,308,160.00$ & $1,308,160.00$ & $1,308,160.00$ & 1,308,160.00 \\
\hline 2020 & $1,308,160.00$ & $1,308,160.00$ & $1,308,160.00$ & $1,308,160.00$ & $1,308,160.00$ \\
\hline 2021 & $1,308,160.00$ & $9,192,960.00$ & $1,308,160.00$ & $1,308,160.00$ & $1,308,160.00$ \\
\hline 2022 & $1,308,160.00$ & $1,308,160.00$ & $17,308,160.00$ & $1,308,160.00$ & $1,308,160.00$ \\
\hline 2023 & $1,308,160.00$ & $1,308,160.00$ & $1,308,160.00$ & $1,308,160.00$ & $1,308,160.00$ \\
\hline 2024 & $1,308,160.00$ & $1,308,160.00$ & $1,308,160.00$ & $1,308,160.00$ & $1,308,160.00$ \\
\hline 2025 & $1,308,160.00$ & $9,192,960.00$ & $1,308,160.00$ & $1,308,160.00$ & $1,308,160.00$ \\
\hline 2026 & $1,308,160.00$ & $1,308,160.00$ & $1,308,160.00$ & $1,308,160.00$ & $1,308,160.00$ \\
\hline 2027 & $1,308,160.00$ & $1,308,160.00$ & $17,308,160.00$ & $1,308,160.00$ & $1,308,160.00$ \\
\hline 2028 & $1,308,160.00$ & $1,308,160.00$ & $1,308,160.00$ & $1,308,160.00$ & $1,308,160.00$ \\
\hline 2029 & $1,308,160.00$ & 9,192,960.00 & $1,308,160.00$ & $1,308,160.00$ & $1,308,160.00$ \\
\hline 2030 & $1,308,160.00$ & $1,308,160.00$ & $1,308,160.00$ & $1,308,160.00$ & $1,308,160.00$ \\
\hline 2031 & $1,308,160.00$ & $1,308,160.00$ & $1,308,160.00$ & $1,308,160.00$ & $1,308,160.00$ \\
\hline 2032 & $1,308,160.00$ & $1,308,160.00$ & $17,308,160.00$ & $1,308,160.00$ & $1,308,160.00$ \\
\hline 2033 & $1,308,160.00$ & $9,192,960.00$ & $1,308,160.00$ & $1,308,160.00$ & $1,308,160.00$ \\
\hline 2034 & $1,308,160.00$ & 1,308,160.00 & $1,308,160.00$ & $1,308,160.00$ & $1,308,160.00$ \\
\hline 2035 & 1,308,160.00 & 1,308,160.00 & $1,308,160.00$ & $1,308,160.00$ & $1,308,160.00$ \\
\hline 2036 & $1,308,160.00$ & 9,192,960.00 & $1,308,160.00$ & 1,308,160.00 & $1,308,160.00$ \\
\hline Total & $26.163 .200,00$ & $73.472 .000,00$ & $90.163 .200,00$ & $86.502 .400,00$ & $56.217 .600,00$ \\
\hline
\end{tabular}

FIGURA 11 - Resumo dos serviços na rodovia (por seção) obtidos do HDM-4

Fonte: Resultado da pesquisa (2018) 
Simultaneamente, o Figura 12 demonstra o desenvolvimento do IRI para cada solução adotada, em relação ao período de análise, confirmando assim, a maior eficácia da solução CREMA 1 quanto as demais alternativas.
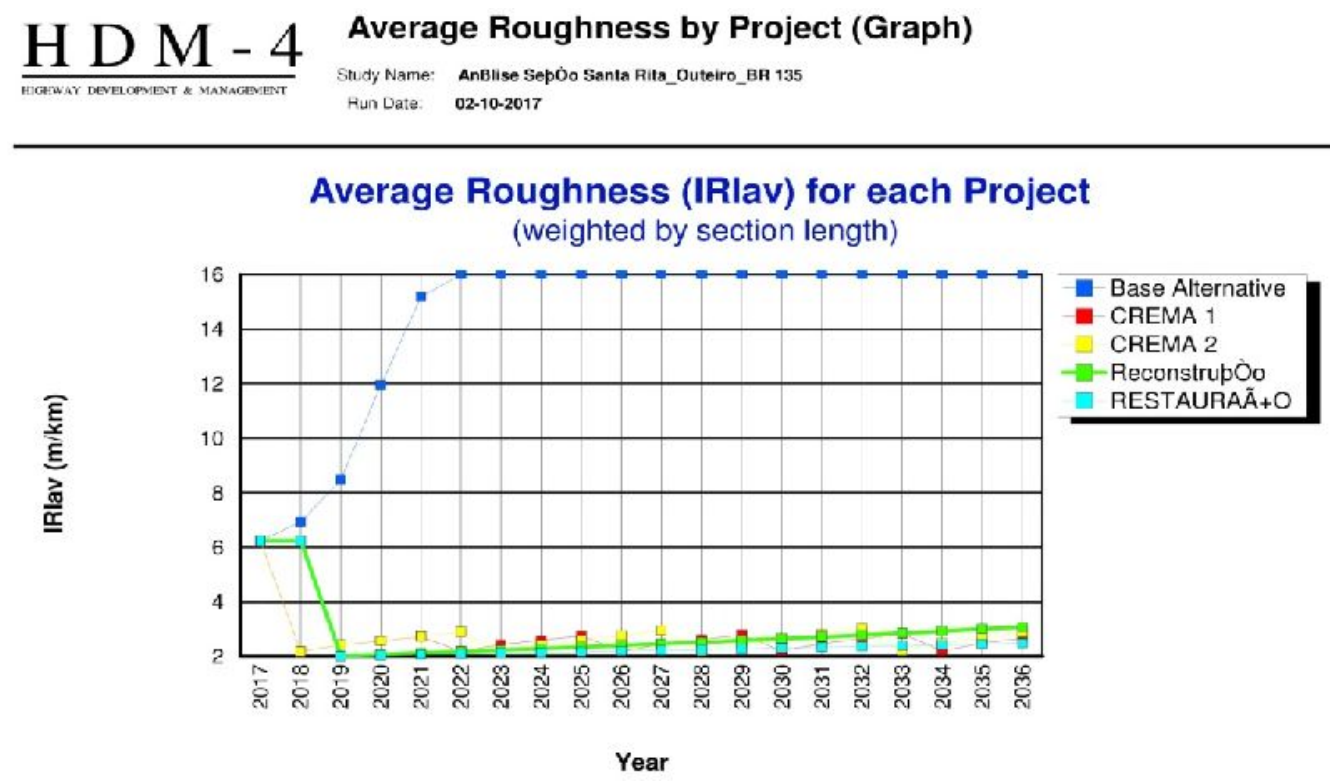

FIGURA 12 - IRI médio por projeto obtido no programa HDM-4

Fonte: Resultado da pesquisa (2018)

Chama-se atenção para fato do presente trabalho utilizar um método simples (coleta/aplicação), porém satisfatório, pois as diversas simulações foram possíveis pela confiabilidade dos dados obtidos in loco. Dessa forma, entende-se que o resumo dos custos totais de transporte, resumo dos serviços na rodovia e IRI médio por projeto possibilitam a gerência dos gatos necessários no decorrer anos.

\section{CONCLUSÃO}

$\mathrm{Na}$ aplicação do Sistema de Gerência de Pavimentos por intermédio da SRMA/DNIT, destaca-se que a maior dificuldade esteve na coleta de informações para o desenvolvimento de um banco de dados que representasse a realidade do trecho em estudo, onde para cada informação obtida, realizou-se um procedimento de investigação, permitindo a aplicação dos dados no programa HDM-4 com objetivo de efetivar uma análise comparativa entre as alternativas de M\&R desde 2017.

De acordo com a avaliação dos resultados obtidos, percebeu-se que o desenvolvimento do SGP é essencial para uma tomada de decisão, pois sua implantação permite maior controle de dados, maior economia aos cofres públicos, conhecer e comparar as possíveis intervenções para um trecho rodoviário, antever custos para um período de tempo predeterminado e, também, a diminuição no custo operacional dos veículos que trafegam na rodovia.

Apesar de tantas vantagens encontradas no SGP, é imprescindível que todos se conscientizem de que sua aplicação tem muito que se desenvolver no território maranhense, porquanto ao verificar os valores obtidos pelo programa HDM-4 é possível notar que a diferença nos custos totais de transporte sem taxa de desconto entre a pior alternativa (Base Alternativa) e a melhor alternativa (CREMA 1) é de aproximadamente $R \$ 7$ bilhões para um período de 20 anos. Lembrando que, individualmente, a conservação rotineira (Base Alternativa) é excessivamente utilizada no Brasil, gerando grande prejuízo aos cofres públicos. 
Em virtude dos fatos mencionados, conclui-se que este estudo em nível de projeto possibilitou a formação de um banco de dados que representa um passo muito importante para o desenvolvimento do SGP no estado do Maranhão. E apesar deste trabalho ser realizado em um nível básico de análise, poderá ser potencializado no decorrer dos anos através de maiores pesquisas que determinarão a continuidade do processo de atualização das informações.

\section{REFERÊNCIAS}

BENNETT, C.R.; PATERSON, W.D.O. Volume five - A guide to calibration and adaptation: The Hightway Development and Management Series. Birmingham: The University of Birmingham, 2000. 229p.

CONSÓRCIO DYNATEST ENGEMAP. Relatório técnico: catálogo de soluções de manutenção para pavimentos flexíveis. 2015. 41p.

CNT - CONFEDERAÇÃO NACIONAL DE TRANSPORTES - Estudo da CNT apresenta as rodovias esquecidas pelo poder público. Confederação Nacional de Transportes. 2018. Disponível em: <https://www.cnt.org.br/agencia-cnt/rodoviasesquecidas-do-brasil>. Acesso em 04/01/2019

CNT - Confederação Nacional de Transportes - Pesquisa CNT de Rodovias 2016 - Relatório Gerencial. Confederação Nacional de Transportes. 2016. Disponível em: $<$ http://pesquisarodovias.cnt.org.br>. Acesso em 10/12/2018.

DNIT - Departamento Nacional de infraestrutura de transportes. Manual de gerência de pavimentos. Rio de Janeiro, 2011. 189p.

FERNANDES, F. M. L. da S. Software de gerenciamento de pavimentos aplicado a vias urbanas de cidades de pequeno a médio porte. Projeto de Graduação. Departamento de Engenharia de Transportes. Curso de Graduação em Engenharia Civil. Universidade Federal do Rio de Janeiro. Rio de Janeiro, 2017. 106 p.

HUDSON, W.R.; HAAS, R.; PERDIGO, R.D. Pavement management system development: national cooperative highway research program, report 215 . Washington, 1979. 32p.

KERALI, H.G.R. Volume one - Overview of HDM-4: ter hightway development and management series. Birmingham: The University os Birmingham, 2000. 43p.

ODA, S. Notas de Aula. Disciplina EER 555 Pavimentação B. Departamento de Engenharia de Transportes. Escola Politécnica da Universidade Federal do Rio de Janeiro - UFRJ, 2014.

ODA, S. Notas de Aula. Disciplina EER 555 Pavimentação A. Departamento de Engenharia de Transportes. Escola Politécnica da Universidade Federal do Rio de Janeiro - UFRJ, 2016.

ZOFKA, A. Proactive pavement asset management with climate change aspects. Warszawa, $2018.2 \mathrm{p} . \quad$ Disponível em: $<$ https://iopscience.iop.org/article/10.1088/1757-899X/356/1/012005>. doi:10.1088/1757-899X/356/1/012005. Acesso em 07/02/2019. 